\title{
DOMÍNIOS AFETADOS NA QUALIDADE DE VIDA DO PACIENTE RENAL CRÔNICO EM TRATAMENTO HEMODIALÍTICO: REVISÃO SISTEMÁTICA
}

\author{
DOMAINS AFFECTED IN THE QUALITY OF LIFE OF RENAL PATIENT IN \\ HEMODIALYTIC TREATMENT: SYSTEMATIC REVIEW
}

\section{DIMENSIÓN AFECTADOS EN LA CALIDAD DE VIDA DEL PACIENTES RENAL CRÓNICO EN TRATAMIENTO HEMODIALÍTICO: REVISIÓN SISTEMÁTICA}

\author{
Renata Alessandra Evangelista ${ }^{1}$, Natália Cristina Pires ${ }^{2}$, Cildiléia Santos Silva ${ }^{3}$, Alexandre \\ Assis Bueno $^{4}$, Luiz Almeida Silva ${ }^{5}$
}

\section{RESUMO}

Objetivo: Sintetizar o conhecimento produzido acerca das publicações na literatura nacional e internacional sobre os domínios afetados na qualidade de vida dos pacientes renais crônicos em tratamento hemodialítico. Métodos: Foi realizada uma revisão sistemática nas bases de dados, LILACS, MEDLINE e CINAHL, nos últimos 10 anos. Resultados: Foram analisados 9 artigos, a maioria dos estudos eram descritivos e transversais, apresentaram evidências sobre as relações das dimensões afetadas na qualidade de vida dos indivíduos em tratamento hemodialítico. Os achados revelaram baixos escores de qualidade de vida, principalmente no domínio físico, sendo o aspecto social aquele de melhor escore. A identificação dos itens impactantes da qualidade de vida dessa população permite, aos profissionais de saúde, repensar a assistência atualmente oferecida, minimizando as consequências negativas decorrentes desse processo. Conclusão: Revela-se que o cuidado dispensado aos pacientes deve englobar estratégias para melhorar a saúde e qualidade de vida dos pacientes.

Descritores: Qualidade de Vida; Insuficiência Renal Crônica; Adulto; Diálise Renal

\footnotetext{
${ }^{1}$ Professor Associado I da UFG da Regional Catalão (RC/UFG), Departamento de Enfermagem. Professor e Orientador do Mestrado Profissional em Gestão Organizacional do RC/UFG. Graduação em Enfermagem pela Escola de Enfermagem de Ribeirão Preto, Mestrado em Enfermagem Fundamental pela USP e Doutorado em Interunidades pela Escola de Enfermagem da USP de Ribeirão Preto.

${ }^{2}$ Acadêmica do Curso de Enfermagem da Universidade Federal de Goiás - Regional Catalão (UFG/RC).

${ }^{3}$ Acadêmica do Curso de Enfermagem da Universidade Federal de Goiás - Regional Catalão (UFG/RC).

${ }^{4}$ Professor Mestre do Curso de Enfermagem da UFG - Regional Catalão (UFG/RC).

${ }^{5}$ Professor Doutor do Curso de Enfermagem da UFG - Regional Catalão (UFG/RC).
} 


\begin{abstract}
Objective: The objective the study was to synthesize the knowledge produced about the publications in the national and international literature on the domains affected in the quality of life of chronic renal patients on hemodialysis. Methods: This is a systematic review was carried out, we surveyed LILACS, MEDLINE and CINAHL published in the last 10 years. Results: A total of the 9 articles were analyzed, a most of the studies were descriptive and cross-section, presenting evidence on the relationships of the individuals undergoing hemodialysis treatment. The results revealed low quality of life scores, mainly in the physical domain, being the social aspect the one with the best score. The identification of the impacting items of the quality of life of this population allows health professionals to rethink the assistance currently offered, minimizing the negative consequences of this process. Conclusion: The review indicates the care given to patients should include strategies to improve the health and quality of life of patients.
\end{abstract}

Descriptors: Quality of life; Chronic renal failure; Adult; Renal dialysis.

\title{
RESUMEN
}

Objetivo: Sintetizar el conocimiento producido sobre las publicaciones en la literatura nacional e internacional sobre los ámbitos afectados en la calidad de vida de los pacientes renais crónicos en tratamiento hemodialítico. Métodos: Se realizó una revisión sistemática en las bases de datos, LILACS, MEDLINE y CINAHL, en los últimos 10 años. Resultados: Se analizaron 9 artículos, la mayoría de los estudios eran descriptivos y transversales, presentaron evidencias sobre las relaciones de las dimensiones afectadas en la calidad de vida de los individuos en tratamiento hemodialítico. Los hallazgos revelaron bajos escores de calidad de vida, principalmente en el dominio físico, siendo el aspecto social el de mejor puntaje. La identificación de los elementos impactantes de la calidad de vida de esa población permite, a los profesionales de salud, repensar la asistencia actualmente ofrecida, minimizando las consecuencias negativas derivadas de ese proceso. Conclusión: Se revela que el cuidado dispensado a los pacientes debe englobar estrategias para mejorar la salud y calidad de vida de los pacientes.

Descriptores: Calidad de Vida; Insuficiencia Renal Crónica; Adulto; Diálisis Renal

\section{INTRODUÇÃO}

A Insuficiência Renal Crônica (IRC) é uma síndrome metabólica, decorrente da perda progressiva, na maioria das vezes lenta, da capacidade excretória renal. ${ }^{1}$ Considerando que a função de excreção de catabólitos é resultante especialmente da filtração glomerular, a IRC consiste na perda da filtração glomerular que pode ser ponderada clinicamente pela medida do "clearance" de creatinina em urina de 24 horas. $^{2,3}$ Assim, a definição da IRC se baseia em adulterações na taxa de filtração glomerular ou com presença de lesão parenquimatosa conservada por pelo menos três meses. ${ }^{2}$ 
Apesar de serem incertos os dados sobre a prevalência da doença renal no Brasil, existem estudos de bases transversais e de coorte que apresentam taxa de $1,5 \%$ da doença autorreferida. ${ }^{4} \mathrm{O}$ mesmo estudo indica a presença de $3 \%$ de hipercreatininemia entre a população. Assim, de acordo com estes dados, de 3 a 6 milhões de adultos, em uma amostra populacional, apresentariam esta doença, sendo que $0,05 \%$ realizam tratamento dialítico, ou seja, 100 mil pacientes. ${ }^{5}$

A taxa de prevalência global do tratamento dialítico tem crescido anualmente, sendo que, em 2016, 39.714 pacientes iniciaram tratamento. Desses, $48 \%$ são da região sudeste, $19 \%$, na região Nordeste, $17 \%$, na região Sul, $10 \%$, na região Centro-Oeste e 5\%, na região Norte. ${ }^{6}$

Alguns fatores podem dificultar ou facilitar a adesão ao tratamento do paciente renal crônico, tais como: apoio familiar; apego à religião/crença; negação e esquiva; resiliência. Estes fatores, de um modo geral, não se apresentam de forma isolada, uma vez que alguns deles se caracterizam como dificuldades inerentes à doença, enquanto que outras se apresentam como consequência das primeiras. $^{5}$

Em seu processo de enfrentamento da doença e dos impactos em seu cotidiano, de um modo geral, os pacientes recorrem principalmente à religião/crença e ao apoio da família. Muitas vezes, se utilizam da negação e esquiva frente às situações de estresse, entretanto, a resiliência se apresentou como um importante recurso de enfrentamento dos problemas iniciais frente à IRC e como uma forma de adaptação às mudanças do cotidiano com o tratamento. ${ }^{1}$

O tratamento consiste na hemodiálise, que durará por toda a sua vida ou até se submeterem a um transplante renal bem-sucedido. ${ }^{6}$ Este procedimento leva a filtração dos líquidos extracorpóreos do sangue feito por uma máquina cognominada dialisador, que supre as funções renais. ${ }^{7}$

O objetivo principal do tratamento hemolítico é tornar mínimos os sintomas, causados pelo mau funcionamento dos rins e proporcionar ao paciente uma melhor qualidade de vida, avaliando se sempre o monitoramento dos níveis plasmáticos de potássio, ureia, sódio e cloretos. ${ }^{8}$

O tratamento da IRC é bastante rigoroso e conduz a mudanças drásticas nos hábitos de vida, impondo restrições e limitações às diversas atividades da vida diárias (AVD). Consequentemente, essa condição produz diversos fatores estressores ao paciente, que podem contribuir para baixa adesão ao tratamento, o que conduz ao aumento significativo de 
complicações e impacto negativo na qualidade de vida. $^{9}$

Deste modo, a qualidade de vida desses pacientes com IRC pode ser avaliada através de instrumentos específicos, como KDQOL, KDQOL-SF, Dialysis Quality of life - DIAQOL), sendo que, a escolha é fundamental para viabilização do estudo, pois o mesmo deve estar alinhado com os objetivos da pesquisa e possua domínios necessários para que sejam medidos na população em estudo. $^{10}$

Diante deste contexto o objetivo do estudo foi sintetizar o conhecimento produzido acerca das publicações na literatura nacional e internacional sobre os domínios afetados na qualidade de vida dos pacientes renal crônico em tratamento hemodialítico.

\section{MÉTODO}

A Revisão sistemática da literatura, tem como objetivo agrupar e sumarizar resultados de pesquisas em relação a um tema ou questão específica. ${ }^{11}$ Para estruturação da revisão, seguimos as etapas: $1^{\mathrm{a}}$ foi a formulação da pergunta de revisão; $2^{\mathrm{a}}$ definição dos critérios de inclusão e exclusão, $3^{\mathrm{a}}$ foram selecionados os artigos da amostra; $4^{\mathrm{a}}$ foi realizada a avaliação crítica da qualidade dos estudos, $5^{a}$ etapa fez-se a interpretação dos resultados e na $6^{\text {a }}$, foi elaborado este artigo com a finalidade de divulgar os resultados obtidos. $^{12}$

Para construção da questão referente à pesquisa da revisão, foi empregada a estratégia PICO (acrônimo para população, intervenção, comparação e desfecho) transformado em descritores/palavraschave, que foram utilizados na busca de evidências científicas. Sendo assim, a pergunta norteadora do estudo foi: "Quais domínios da qualidade de vida foram mais afetadas em pacientes renais crônicos submetidos ao tratamento hemodialítico?

Para a busca dos artigos, foram utilizadas as seguintes bases de dados: Medical Literature Analysis and Retrieval System Online (Medline), Literatura Latino-Americana e do Caribe em Ciências da Saúde (LILACS), e Cumulative Index to Nursing and Allied Health Literature (CINAHL).

Os descritores utilizados em português, inglês e espanhol, foram: qualidade de vida (quality of life), insuficiência renal crônica (renal insufficiency chronic), diálise renal (renal dialysis), que fazem parte dos Descritores em Ciências da Saúde - DeCS e Medical Subject Headings - MeSH, além das palavras-chaves relacionadas.

Os critérios de inclusão adotados para este estudo foram: estudos primários 
com pacientes adultos (acima de 18 anos) em tratamento hemodiálitco, com abordagem quantitativa, disponíveis nas bases de dados, publicados em português, inglês e espanhol, que trouxesse informações para responder à pergunta de investigação, não havendo limitações para período de busca e artigos completos. Excluímos deste estudo os artigos que não estavam disponíveis na íntegra, livros, capítulos de livros, relatórios técnicos, cartas e editoriais. Quanto aos artigos que se repetiram foram mantidos aqueles que se encontravam em uma versão mais completa. $^{13}$

Embora o objetivo tenha sido realizar uma revisão sistemática da literatura, optou-se por utilizar como estratégia o guia Preferred Reporting Items for Systematic Reviews and Meta-Analysis (PRISMA). ${ }^{11}$ Este instrumento possibilita um olhar mais amplo sobre a sintetização dos diferentes tipos de evidências por meio de um processo rigoroso para a realização desta avaliação crítica, bem como a garantia do rigor da revisão sistemática. Os dados foram coletados e selecionados com a busca e leitura dupla busca-cega, com um terceiro revisor para a seleção final. A avaliação crítica dos estudos utilizou a lista de verificação Critical Appraisal Checklist for Analytical Cross Sectional Studies disponibilizada pelo The Joanna Briggs Institute- JBI. $^{12}$

Os resultados são apresentados de forma descritiva com o objetivo de sumarizar as evidências com relação as dimensões afetadas na qualidade de vida dos indivíduos em tratamento hemodialítico.

\section{RESULTADOS}

Inicialmente, foram identificados 1.934 artigos potencialmente relevantes para o questionamento levantado. Desse número, 194 atenderam aos critérios de inclusão e foram lidos na íntegra (Figura 1) e avaliados a partir da questão norteadora da revisão. Após a leitura, 09 artigos foram incluídos neste estudo por apresentarem evidências sobre as relações das dimensões afetadas na qualidade de vida dos indivíduos em tratamento hemodialítico (Quadro 1). A partir da descrição dos artigos do estudo, foram identificados estudos realizados em clinicas e hospitais que continham a instalação da hemodiálise para os pacientes. 
Figura1. Diagrama da seleção de artigos para revisão sistemática.

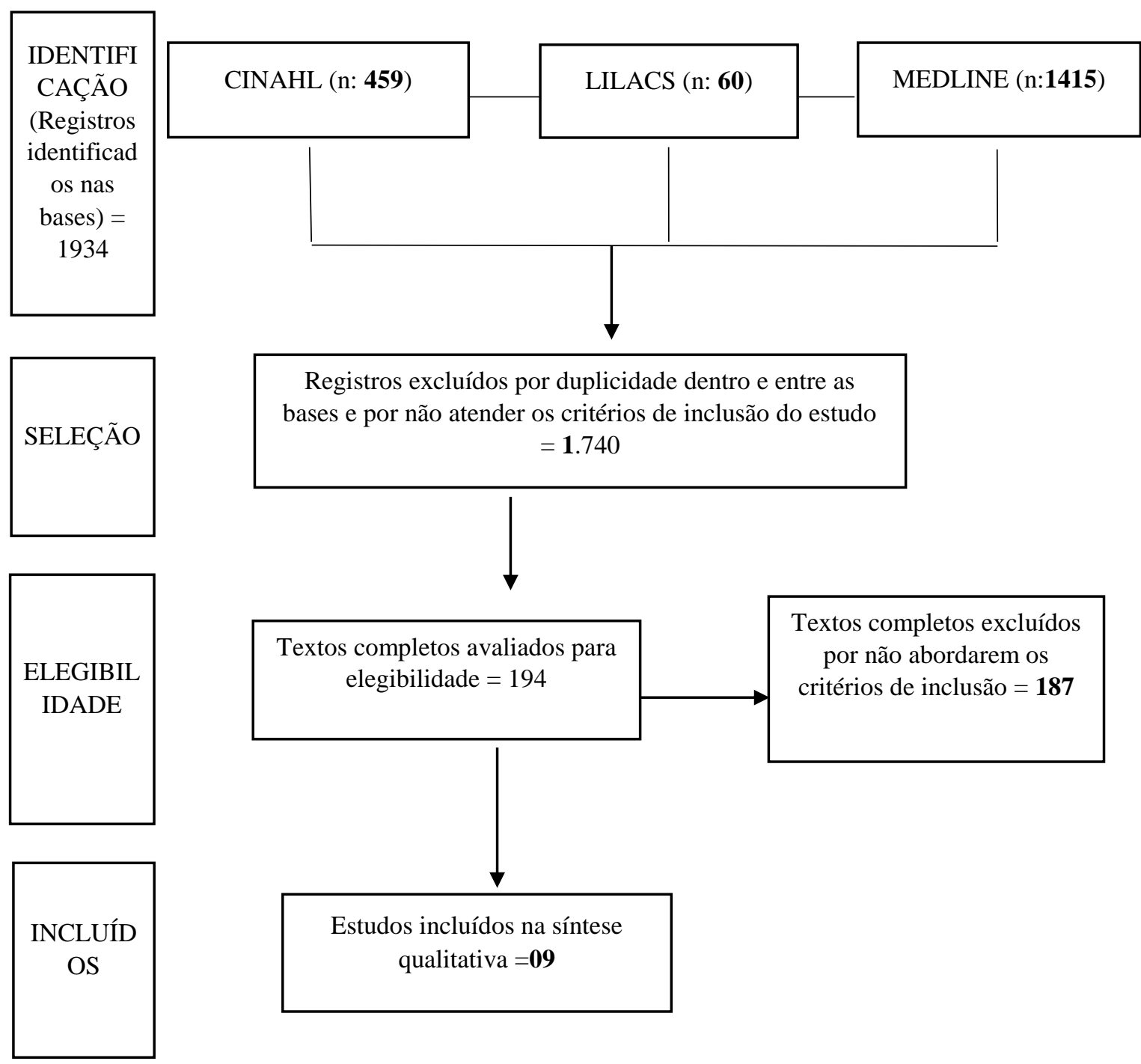


Quadro 1: Distribuição dos artigos selecionados conforme autor, base de dados, país, revista, delineamento do estudo, instrumentos utilizados resultados e principais conclusões - 2008 a 2017.

\begin{tabular}{|c|c|c|c|c|c|c|c|}
\hline AUTORES & $\begin{array}{c}\text { BASE DE } \\
\text { DADOS/ } \\
\text { PAÍS DE } \\
\text { ORIGEM/ } \\
\text { ANO DE } \\
\text { PUBLICAÇÃO } \\
\end{array}$ & $\begin{array}{c}\text { DELINEAMENTO } \\
\text { DO } \\
\text { ESTUDO }\end{array}$ & REVISTA & $\begin{array}{l}\text { OBJETIVO DO } \\
\text { ESTUDO }\end{array}$ & $\begin{array}{l}\text { INSTRUMEN } \\
\text { TOS }\end{array}$ & RESULTADOS & SÍNTESE DA CONCLUSÃO \\
\hline $\begin{array}{l}\text { Guerra- } \\
\text { Guerrero, V; } \\
\text { Sanhueza- } \\
\text { Alvarado, O; } \\
\text { Cáceres-Espina, } \\
\text { M. }\end{array}$ & $\begin{array}{l}\text { LILACS } \\
\text { Chile } \\
2012\end{array}$ & $\begin{array}{l}\text { Estudo de } \\
\text { correlação, } \\
\text { exploratória, } \\
\text { descritiva e } \\
\text { transversal }\end{array}$ & $\begin{array}{c}\text { Rev. } \\
\text { Latino-Am. } \\
\text { Enfermage } \\
\text { m }\end{array}$ & $\begin{array}{l}\text { Determinar a } \\
\text { qualidade de vida } \\
\text { de pessoas em } \\
\text { hemodiálise crônica } \\
\text { e sua relação com } \\
\text { variáveis } \\
\text { sociodemográficas, } \\
\text { médico-clínicas e } \\
\text { de laboratório. }\end{array}$ & KDQOL -SF & $\begin{array}{l}\text { Os resultados da QV } \\
\text { revelaram as notas mais } \\
\text { baixas nos componentes } \\
\text { físico, mental e psicossocial. } \\
\text { As subescalas } \\
\text { sintomas/problemas (S) e } \\
\text { efeitos da doença (E), pelo } \\
\text { contrário, mostraram as notas } \\
\text { médias mais altas. }\end{array}$ & $\begin{array}{l}\text { Concluíram que, o perfil } \\
\text { sociodemográfico, junto com as } \\
\text { características médico-clínicas e de } \\
\text { laboratório dos pacientes sob } \\
\text { tratamento dialítico, são aspectos que } \\
\text { contribuem para o baixo nível de QV } \\
\text { relacionados a idade mais alta, baixa } \\
\text { escolaridade, baixa renda, duração } \\
\text { do tratamento, hospitalizações e } \\
\text { ausência de transplantes. }\end{array}$ \\
\hline $\begin{array}{c}\text { Kusumota, L.; } \\
\text { Marques, S; } \\
\text { Haas,VJ.; } \\
\text { Rodrigues, RAP.; }\end{array}$ & $\begin{array}{c}\text { LILACS } \\
\text { Brasil } \\
2008\end{array}$ & $\begin{array}{l}\text { Estudo seccional e } \\
\text { populacional }\end{array}$ & $\begin{array}{c}\text { Acta } \\
\text { Paulista de } \\
\text { Enfermagem }\end{array}$ & $\begin{array}{l}\text { Caracterizar a } \\
\text { população de } \\
\text { pacientes adultos e } \\
\text { idosos em } \\
\text { hemodiálise } \\
\text { residentes em } \\
\text { Ribeirão Preto-SP e } \\
\text { avaliar e descrever } \\
\text { as diferenças da } \\
\text { QVRS destes } \\
\text { pacientes. }\end{array}$ & KDQOL-SF & $\begin{array}{l}\text { Os pacientes referiram } \\
\text { complicações físicas } \\
\text { relacionadas à IRCT e ao } \\
\text { tratamento por hemodiálise, } \\
\text { destacando-se: anemia, } \\
\text { cãibras, fraqueza, dor, } \\
\text { hipotensão arterial durante a } \\
\text { hemodiálise e prurido, dentre } \\
\text { outras. }\end{array}$ & $\begin{array}{l}\text { Concluiu que a comparação da QVRS } \\
\text { de adultos e idosos é importante para } \\
\text { avaliar o impacto da doença e do } \\
\text { tratamento nas condições de vida e, } \\
\text { desta forma, direcionar a assistência a } \\
\text { esses pacientes, de forma individual e } \\
\text { coletiva. O KDQOL-SF mostrou-se } \\
\text { um instrumento capaz de avaliar a } \\
\text { QVRS de pacientes adultos e idosos } \\
\text { em hemodiálise. }\end{array}$ \\
\hline $\begin{array}{l}\text { Marinho, CLA.; } \\
\text { Oliveira,JF.de.; } \\
\text { Borges,JE.da.S.; } \\
\text { Silva, RS.da.; } \\
\text { Fernandes, } \\
\text { FECV }\end{array}$ & $\begin{array}{l}\text { CINAHL } \\
\text { Brasil } \\
2017\end{array}$ & $\begin{array}{l}\text { Estudo descritivo, } \\
\text { transversal }\end{array}$ & $\begin{array}{l}\text { Revista } \\
\text { Rene }\end{array}$ & $\begin{array}{l}\text { Analisar a qualidade } \\
\text { de vida de pessoas } \\
\text { com doença crônica }\end{array}$ & KDQOL-SF & $\begin{array}{l}\text { As dimensões mais afetadas } \\
\text { estão relacionadas ao status de } \\
\text { trabalho e à função física. Os } \\
\text { domínios com as maiores } \\
\text { médias foram: Função Sexual, } \\
\text { Função Cognitiva, função } \\
\text { social e dor. }\end{array}$ & $\begin{array}{l}\text { Concluíram que a doença renal } \\
\text { crônica e o tratamento de hemodiálise } \\
\text { interferem na qualidade de vida dos } \\
\text { indivíduos, com maiores perdas na } \\
\text { situação do trabalho e função física, e } \\
\text { uma melhor repercussão nos domínios } \\
\text { da dor, função social, função } \\
\text { cognitiva e função sexual. }\end{array}$ \\
\hline
\end{tabular}




\begin{tabular}{|c|c|c|c|c|c|c|c|}
\hline $\begin{array}{l}\text { Cunha, MS.; } \\
\text { Andrade, V.; } \\
\text { Guedes, CAV.; } \\
\text { Meneghetti, } \\
\text { CHZ.; Aguiar, } \\
\text { AP; Cardoso, AL }\end{array}$ & $\begin{array}{c}\text { LILACS } \\
\text { Brasil } \\
2009\end{array}$ & $\begin{array}{l}\text { Estudo descritivo, } \\
\text { transversal }\end{array}$ & $\begin{array}{l}\text { Fisioterapia } \\
\text { e Pesquisa, } \\
\text { São Paulo }\end{array}$ & $\begin{array}{l}\text { Avaliar a capacidade } \\
\text { funcional e a } \\
\text { qualidade de vida } \\
\text { em pacientes com } \\
\text { incapacidade renal } \\
\text { crônica (IRC) } \\
\text { submetidos a } \\
\text { tratamento } \\
\text { hemodialítico e } \\
\text { verificar possíveis } \\
\text { correlações entre } \\
\text { essas variáveis } \\
\text { clínicas e idade, } \\
\text { índice de massa } \\
\text { corpórea (IMC) e } \\
\text { tempo de } \\
\text { hemodiálise. }\end{array}$ & SF 36 & $\begin{array}{l}\text { A capacidade funcional dos } \\
\text { pacientes com IRC } \\
\text { submetidos a tratamento } \\
\text { hemodialítico mostrou-se } \\
\text { abaixo dos valores preditos } \\
\text { quanto à distância percorrida } \\
\text { no TC6' e à força dos } \\
\text { músculos respiratórios } \\
\text { (principalmente musculatura } \\
\text { expiratória), tendo relatado } \\
\text { em média sensação de fadiga } \\
\text { leve }\end{array}$ & $\begin{array}{l}\text { Dor e prejuízo na vitalidade foram } \\
\text { indicados como os fatores que mais } \\
\text { interferem em sua qualidade de vida } \\
\text { relacionada à saúde, tendo fatores } \\
\text { como idade, índice de massa corporal } \\
\text { e o tempo de hemodiálise não se } \\
\text { mostrado relevantes na maioria dos } \\
\text { domínios desta. }\end{array}$ \\
\hline $\begin{array}{l}\text { Mortari, DM.; } \\
\text { Menta, M.; } \\
\text { Scapini, KB.; } \\
\text { Rockembach, } \\
\text { CWF.; Duarte, } \\
\text { A; Leguisamo, } \\
\text { CP }\end{array}$ & $\begin{array}{c}\text { LILACS } \\
\text { Brasil } \\
2010\end{array}$ & $\begin{array}{l}\text { Estudo descritivo, } \\
\text { transversal }\end{array}$ & $\begin{array}{l}\text { Scientia } \\
\text { Medica } \\
\text { (Porto } \\
\text { Alegre) }\end{array}$ & $\begin{array}{l}\text { Avaliar a qualidade } \\
\text { de vida de } \\
\text { indivíduos com } \\
\text { doença renal crônica } \\
\text { terminal submetidos } \\
\text { à hemodiálise. SLEpp: }\end{array}$ & SF 36 & $\begin{array}{l}\text { Os maiores } \\
\text { comprometimentos foram } \\
\text { encontrados nos aspectos } \\
\text { físicos e no estado geral de } \\
\text { saúde. Os indivíduos } \\
\text { apresentaram valores } \\
\text { intermediários do escore de } \\
\text { qualidade de vida nos } \\
\text { domínios vitalidade, dor e } \\
\text { saúde mental. O menor } \\
\text { comprometimento foi } \\
\text { encontrado no domínio } \\
\text { aspectos sociais. }\end{array}$ & $\begin{array}{l}\text { Verificaram no presente estudo que, a } \\
\text { amostra de pacientes com doença } \\
\text { renal crônica terminal submetidos à } \\
\text { hemodiálise, tanto nos homens quanto } \\
\text { nas mulheres, houve redução nos } \\
\text { escores de qualidade de vida, } \\
\text { principalmente nos domínios estado } \\
\text { geral de saúde e limitação por } \\
\text { aspectos físicos. }\end{array}$ \\
\hline $\begin{array}{c}\text { Grasselli, CSM.; } \\
\text { Chaves, E.C; } \\
\text { Simão, TP.; } \\
\text { Botelho, PB.; } \\
\text { Silva, RR. }\end{array}$ & $\begin{array}{c}\text { LILACS } \\
\text { Brasil } \\
2012\end{array}$ & $\begin{array}{l}\text { Estudo descritivo, } \\
\text { transversal }\end{array}$ & $\begin{array}{c}\text { Revista } \\
\text { Brasileira } \\
\text { Clinica } \\
\text { Medica São } \\
\text { Paulo }\end{array}$ & $\begin{array}{l}\text { Avaliar a QV dos } \\
\text { pacientes } \\
\text { submetidos à } \\
\text { hemodiálise. }\end{array}$ & KDQOL-SF & $\begin{array}{l}\text { A média dos escores } \\
\text { encontrada nas dimensões } \\
\text { papel profissional e função } \\
\text { física, as quais englobam os } \\
\text { possíveis problemas que o } \\
\text { paciente pode apresentar em } \\
\text { relação ao trabalho ou outras } \\
\text { atividades habituais devido à }\end{array}$ & $\begin{array}{l}\text { Neste estudo, foi observado que a QV } \\
\text { dos pacientes foi afetada } \\
\text { negativamente pelas dimensões de } \\
\text { papel profissional, vitalidade (energia } \\
\text { e fadiga), função física, função } \\
\text { emocional, saúde geral e sobrecarga } \\
\text { da doença renal na vida dos pacientes; } \\
\text { o que pode ser atribuído às }\end{array}$ \\
\hline
\end{tabular}




\begin{tabular}{|c|c|c|c|c|c|c|c|}
\hline & & & & & & $\begin{array}{l}\text { saúde físicas foram as mais } \\
\text { baixas. Ao analisar as médias } \\
\text { das dimensões energia/fadiga, } \\
\text { sobrecarga da doença renal na } \\
\text { vida dos pacientes, saúde } \\
\text { geral e função emocional, na } \\
\text { dimensão dos efeitos da } \\
\text { doença renal sobre as } \\
\text { condições da vida diária, em } \\
\text { que a média que prevaleceu } \\
\text { nessa dimensão foi de } \\
\text { (62,37\%), assim como para as } \\
\text { dimensões do sono, dor, } \\
\text { funcionamento físico, bem } \\
\text { estar emocional, função } \\
\text { sexual a média foi (76,036\%). }\end{array}$ & $\begin{array}{l}\text { dificuldades e desafios provocados } \\
\text { pela doença renal e seu tratamento. } \\
\text { Por outro lado, os altos escores } \\
\text { encontrados para as dimensões } \\
\text { satisfação do paciente em relação ao } \\
\text { apoio recebido por familiares e } \\
\text { amigos (tempo que passa na presença } \\
\text { destes), vida sexual, função cognitiva, } \\
\text { dor, sono, suporte social, lista de } \\
\text { sintomas/problemas, efeitos da } \\
\text { doença renal, estímulo pela equipe de } \\
\text { diálise, funcionamento físico, bem } \\
\text { estar emocional, função social e } \\
\text { qualidade de interação social foram } \\
\text { dimensões que tiveram altos níveis de } \\
\text { escores e contribuíram positivamente } \\
\text { com a avaliação da QV. }\end{array}$ \\
\hline $\begin{array}{l}\text { Kharame, ZT.; } \\
\text { Zamanian, H; } \\
\text { Foroozanfar, S; } \\
\text { Afsahi, S. }\end{array}$ & $\begin{array}{l}\text { MEDLINE } \\
\text { Iran } \\
2014\end{array}$ & $\begin{array}{l}\text { Estudo descritivo } \\
\text { transversal }\end{array}$ & $\begin{array}{l}\text { Global } \\
\text { Journal of } \\
\text { Health } \\
\text { Science }\end{array}$ & $\begin{array}{l}\text { Determinar a relação } \\
\text { entre bem-estar } \\
\text { espiritual e } \\
\text { qualidade de vida } \\
\text { em pacientes em } \\
\text { hemodiálise. }\end{array}$ & SF 36 & $\begin{array}{l}\text { Escores mais altos e mais } \\
\text { baixos foram vistos para o } \\
\text { funcionamento social e } \\
\text { limitações de função devido a } \\
\text { problemas de saúde física. Os } \\
\text { resultados do modelo de } \\
\text { regressão logística múltipla } \\
\text { mostraram que o bem-estar } \\
\text { religioso se associou a melhor } \\
\text { QV nos domínios físico e } \\
\text { mental. }\end{array}$ & $\begin{array}{l}\text { Os achados destacam a importância } \\
\text { do bem-estar espiritual para a QV dos } \\
\text { pacientes em hemodiálise. O bem- } \\
\text { estar religioso predisse melhor } \\
\text { qualidade de vida em pacientes. O } \\
\text { foco no reforço da espiritualidade no } \\
\text { atendimento rotineiro aos pacientes } \\
\text { poderia melhorar os diferentes } \\
\text { aspectos da QV. Além disso, o apoio } \\
\text { espiritual da família ou de instituições } \\
\text { de caridade pode ter um bom impacto } \\
\text { na qualidade de vida desses pacientes. } \\
\text { Em relação à alta correlação entre } \\
\text { bem-estar religioso e bem-estar } \\
\text { espiritual, conclui-se a base religiosa } \\
\text { das crenças existenciais. }\end{array}$ \\
\hline $\begin{array}{c}\text { Silva, KAL; } \\
\text { Cargnin, MCS; } \\
\text { Ventura,J; Paula, } \\
\text { SF; Groos, JV }\end{array}$ & $\begin{array}{c}\text { LILACS } \\
\text { Brasil } \\
2017\end{array}$ & $\begin{array}{l}\text { Estudo descritivo } \\
\text { transversal }\end{array}$ & $\begin{array}{l}\text { Revista de } \\
\text { Enfermagem } \\
\text { REUOL on } \\
\text { line }\end{array}$ & $\begin{array}{l}\text { Avaliar a qualidade } \\
\text { de vida de pessoas } \\
\text { com Insuficiência } \\
\text { Renal Crônica em } \\
\text { tratamento }\end{array}$ & KDQOL-SF & $\begin{array}{l}\text { As dimensões do KDQOL- } \\
\text { SFTM com menores escores } \\
\text { foram: função emocional, } \\
\text { função física, sobrecarga da } \\
\text { doença renal e papel }\end{array}$ & $\begin{array}{l}\text { Constatou-se que a qualidade de vida } \\
\text { relacionada à saúde destes pacientes } \\
\text { encontra-se comprometida em } \\
\text { diversos aspectos avaliados pelo } \\
\text { KDQOL-SF 1.3. O papel profissional }\end{array}$ \\
\hline
\end{tabular}

Rev Enferm Atenção Saúde [Online]. Out/Dez 2018; 7(3):150-164

ISSN 2317-1154 


\begin{tabular}{|c|c|c|c|c|c|c|c|}
\hline & & & & $\begin{array}{l}\text { hemodialítico por } \\
\text { meio do instrumento } \\
\text { KDQOL-SF1.3 }\end{array}$ & & $\begin{array}{l}\text { profissional. Maiores escores } \\
\text { foram: função social, dor, } \\
\text { estímulo da equipe de diálise } \\
\text { e função cognitiva. }\end{array}$ & $\begin{array}{l}\text { apresentou o pior escore, seguido pela } \\
\text { função física, função emocional e } \\
\text { sobrecarga da doença renal. Por outro } \\
\text { lado, houve resultados satisfatórios } \\
\text { nas dimensões estímulo da equipe de } \\
\text { diálise, função cognitiva, função } \\
\text { social e dor, que contribuíram para a } \\
\text { QVRS da população estudada. }\end{array}$ \\
\hline $\begin{array}{l}\text { Stockschneider, } \\
\text { FBM; Imhof, S; } \\
\text { Silva, RMGS; } \\
\text { Baggio, FB; Luz } \\
\text { Filho, HA; } \\
\text { Vieira, MA }\end{array}$ & $\begin{array}{c}\text { LILACS } \\
\text { Brasil } \\
2017\end{array}$ & $\begin{array}{l}\text { Estudo descritivo } \\
\text { transversal }\end{array}$ & $\begin{array}{l}\text { Saúde e } \\
\text { Ambiente } \\
\text { (Revista } \\
\text { Interdiscipli } \\
\text { nar) }\end{array}$ & $\begin{array}{l}\text { Avaliar as mudanças } \\
\text { na Qualidade de } \\
\text { Vida }(Q V) \text { de } \\
\text { pacientes } \\
\text { submetidos ao } \\
\text { tratamento } \\
\text { hemodialítico, em } \\
\text { um período de } 4 \\
\text { anos. }\end{array}$ & KDQOL-SF & $\begin{array}{l}\text { Na avaliação de } 2013 \text {, } \\
\text { maiores escores foram obtidos } \\
\text { em estímulo da equipe e } \\
\text { satisfação do paciente, } \\
\text { enquanto que menores escores } \\
\text { foram observados nos } \\
\text { domínios funcionamento } \\
\text { físico, saúde geral e função } \\
\text { social. O escore de QV total } \\
\text { foi semelhante nos dois } \\
\text { períodos de avaliação. }\end{array}$ & $\begin{array}{l}\text { O presente estudo mostrou que a QV } \\
\text { geral dos pacientes se manteve entre } \\
\text { os anos de } 2009 \text { e 2013, porém, nos } \\
\text { pacientes idosos houve uma } \\
\text { diminuição significativa da QV geral } \\
\text { durante o período estudado } \\
\text { influenciado por aspectos físicos e } \\
\text { sociais. }\end{array}$ \\
\hline
\end{tabular}




\section{DISCUSSÃO}

Ao observar os estudos selecionados, algumas características comuns se destacam e podem contribuir para fundamentar ações que abordem a temática em questão.

A busca nas bases de dados foi realizada sem qualquer tipo de filtro, mesmo assim os nove estudos selecionados foram publicados nos últimos nove anos. Tal achado indica uma preocupação recente com a qualidade de vida de pacientes em tratamento hemodialítico e, consequentemente, escassez da literatura especializada. Ressalta-se que em dois artigos as populações estudadas foram de outros países, Chile e Iran. ${ }^{14,15}$

Outro fator que pode comprometer a qualidade da evidência para fundamentar a prática, é o tipo de método predominante. Nesta busca, sete, dos artigos encontrados, foram identificados como transversais. $\mathrm{O}$ estudo transversal, possui a característica de descrever um momento específico e não é o tipo de pesquisa indicado para sugerir algum tipo de associação ou relação entre as variáveis observadas e constituem estudos de evidências fracas. ${ }^{16}$ Acrescentase ainda, que não foi encontrado algum estudo com intervenção. Todos se limitaram ao diagnóstico situacional.

Por outro lado, seis estudos utilizaram como instrumento de pesquisa o
KDQOL- Kidney Disease Quality of Life. Este instrumento é específico para a verificação da qualidade de vida em pacientes submetidos ao tratamento hemodialítico. Assim, esta revisão apresenta que o KDQOL, além de ter sido traduzido e validado para diversas localidades, ainda conta com o consenso de diversos pesquisadores da área, sendo um indicador para a adoção de seu uso em estudos desta natureza.

Sendo assim, avaliaram-se os domínios mais afetados nos pacientes, esses domínios eram oito na escala KDQOL-SF que avaliavam funcionamento físico (PF), papel-físico (RP), dor corporal (BP), saúde geral (GH), vitalidade (VT), funcionamento social (SF), papelemocional (RE) e saúde mental (MH). ${ }^{1,4,6,14,17,18}$

O questionário SF-36, Medical Outcomes Study 36 - Item Short-Form Health Survey, foi utilizado em 03 estudos. Trata-se de um instrumento genérico de avaliação de qualidade de vida, composto de 36 itens, compostos pelas seguintes dimensões: capacidade funcional, aspectos físicos, dor, estado geral de saúde, vitalidade, aspectos sociais e aspectos emocionais. $^{19-21}$

As mensurações de qualidade de vida podem oferecer dados importantes sobre as dimensões psicológicas e sociais, que estão 
afetando o doente renal crônico. Entretanto, mesmo com a melhora e busca por qualidade nos atendimentos em saúde, aumento da expectativa de vida e de sobrevida dos pacientes, avanços tecnológicos no tratamento, evidências científicas, foi identificado redução da qualidade de vida dos pacientes renais crônicos e as implicações da hemodiálise no cotidiano dos pacientes. De um modo geral, uma das dimensões mais afetadas na qualidade de vida, está o domínio físico e psicológico. $^{22}$

Estudos mundiais, ressaltam nos seus achados um menor funcionamento físico em pacientes com estagio final da doença renal, bem como, uma diminuição exacerbada na escala de componente físico do SF-36 em pacientes submetidos à hemodiálise (HD) e à diálise peritoneal (DP). ${ }^{23}$

Todos os artigos deste estudo, demonstraram o comprometimento da qualidade de vida dos pacientes submetidos a hemodiálise, sendo os domínios físico e cognitivo mais afetados. Todavia, os pacientes com insuficiência renal crônica, contendo os sintomas físicos de fadiga e perda de energia além de restrição da vida social, tiveram os domínios referentes a funcionamento físico, dor corporal e funcionamento social, mensurado no KDQOL-SF com escores mais baixos. ${ }^{18-20}$

Os autores, Kalender; Ozdemir; Dervisoglu; Ozdemir ${ }^{24}$, relatam que, o comprometimento relativo à qualidade de vida em pacientes com diálise peritoneal ambulatorial contínua pode ser explicado por fatores responsáveis pelo tratamento, necessitando de ser dialisado "diariamente" e havendo complicações médicas que podem estar associadas, como a peritonite.

No entanto no estudo de Ikonomou et $a l^{25}$, ocorreu uma diferença quanto a dimensão física, vitalidade e dores corporais entre os grupos de pacientes, evidenciando uma influência entre a idade e a diálise, com a pontuação física inversamente proporcional aos escores do domínio físico.

Embora os pacientes com doença renal crônica necessite de terapias de reposição renal, as modificações nos hábitos de vida e cuidados com a saúde são frequentes. Assim, foi verificada nos estudos uma prevalência de ansiedade, depressão, suporte social percebido, fadiga, indícios de estresse afetando os níveis de qualidade de vida em pacientes. ${ }^{18,20}$ Ainda que o objetivo deste estudo não tenha sido avaliar a qualidade de vida dos familiares e/ou cuidadores, um estudo que avaliou a qualidade de vida dos pacientes renais crônicos e seus cuidadores, os dados foram 
semelhantes, apresentando variáveis de pior pontuação nos cuidadores. ${ }^{26}$

Fica evidente, através desses estudos, que o paciente, ao ser informado da condição de portador da doença crônica, necessitando de tratamento dialítico, tendo a obrigação de frequentar centros de diálise, restrições hídricas e alimentares, além de alterações na jornada de trabalho e na vida social, gera uma instabilidade emocional nos mesmos. Muitas vezes os pacientes passam a conviver com as perdas que vão muito além da função renal, e que o apoio familiar é essencial. ${ }^{27}$

Alguns artigos apontam que quanto maior o tempo de tratamento, menor serão os escores relacionados as atividades diárias, e que, os pacientes com mais de 60 anos possuem mais dificuldades para realizar atividades físicas e de lazer. ${ }^{28}$ Demostraram também, que o sexo feminino foi identificado com mais incapacidade de realizar as atividades físicas e as atividades básicas de vida diária (ABVD), visto que possuem menor qualidade de sono e pior funcionamento sexual. $^{29}$

\section{REFERÊNCIAS}

1. Stockschneider FBM, Imhof $S$, Silva RMGS, Nerbass FB, Luz Filho HA, Vieira MA. Qualidade de vida dos pacientes renais crônicos em um

\section{CONCLUSÕES}

Apesar do crescimento das publicações sobre o impacto da qualidade de vida de adultos com IRC, a temática ainda é pouco explorada. Contudo, foi possível verificar que as dimensões mais afetadas, nesses pacientes, são o domínio físico e capacidade funcional, o que justifica os baixos níveis de vitalidade e problemas relacionados à saúde mental.

A identificação de aspectos impactantes da qualidade de vida dessa população permite, aos profissionais de saúde, repensar a assistência atualmente oferecida, com vistas a auxiliá-los, e assim, minimizar as consequências negativas decorrentes desse processo.

Acredita-se que o cuidado dispensado aos pacientes deve considerar aspectos socioeconômicos e culturais que possibilite uma melhor qualidade de vida a essas pessoas.

Espera-se que esse conhecimento contribua para que esses pacientes se adaptem melhor às suas próprias mudanças e criem suas próprias ferramentas internas de enfrentamento das limitações impostas pela condição crônica.

intervalo de 4 anos. Saúde \& Meio Ambiente. 2017; 6(2):53-64.

2. Draibe JT, Ajzen PG. Manual de diálise. 4ed. Rio de Janeiro: Guanabara; 2010 . 
3. Bastos MG, Kirstajn GM. Doença renal crônica: importância do diagnóstico precoce, encaminhamento imediato e abordagem interdisciplinar estruturada para melhora do desfecho em pacientes ainda não submetidos à diálise. J Bras Nefrol. 2004; 33(01):93-108.

4. Marinho AWGB, Penha AP, Silva MT, Galvão TF. Prevalência de doença renal crônica em adultos no Brasil: revisão sistemática da literatura. Cad Saúde Colet. 2017; 25(3):379-8.

5. Sesso RC, Lopes AA, Thomé FS, Lugon JR, Martins CT. Inquérito brasileiro de diálise crônica 2016. J Bras Nefrol. 2017; 39(3):261-6.

6. Silva RAR, Souza Neto VL, Oliveira GJN, Silva BCO, Rocha CCT, Holanda JRR. Estratégias de enfrentamento utilizadas por pacientes renais crônicos em tratamento hemodialítico. Esc Anna Nery Rev Enferm. 2016; 20(1):147-4.

7. Madeiro AC, Machado PDLC, Bonfim IM, Braqueais AR, Lima FET. Adesão de portadores de insuficiência renal crônica ao tratamento de hemodiálise. Acta Paulista Enferm. 2010; 23:546-51.

8. Cavalcante FA, Saar GQ, Ramos LS, Lima AAM. O uso lúdico em hemodiálise: buscando novas perspectivas na qualidade de atendimento ao paciente no centro de diálise. Rev Eletrônica Facimed. 2011; 3:371- 4.

9. Ministério da Saúde (Brasil), Secretaria de Atenção à Saúde, Departamento de Atenção Especializada e Temática. Diretrizes clínicas para o cuidado ao paciente com doença renal crônica DRC no Sistema Único de Saúde [Internet]. Brasília, DF; 2014 [citado em 13 maio 2018]. Disponível em:

http://bvsms.saude.gov.br/bvs/publicaco es/diretrizes_clinicas_cuidado_paciente _renal.pdf

10. Fleck MPA, organizador. A avaliação da qualidade de vida: guia para profissionais de saúde. Porto Alegre. Artmed; 2008.
11. Mendes KDS, Silveira RCCP, Galvão CM. Revisão Integrativa: método de pesquisa para a incorporação de evidências na saúde e na enfermagem. Texto \& Contexto Enferm. 2008; 17(4):758-64.

12. Aromataris E, Munn Z, editores. Joanna Briggs Institute reviewer's manual [Internet]. Adelaide, Au: The Joanna Briggs Institute; 2017 [citado em 23 abr 2018]. Disponível em: https://reviewersmanual.joannabrig gs.org/

13. Assis DC, Resende DV, Marziale MHP. Associação entre turnos de trabalho, níveis de cortisol salivar, estresse e fadiga em enfermeiros: revisão integrativa. Esc Anna Nery Rev Enferm. [Internet]. 2018 [citado em 21 maio 2018]; 22(1):e20170125.

Disponível em: http://www.scielo.br/pdf/ean/v22n1/pt 1414-8145-ean-2177-9465-EAN-20170125.pdf

14.Guerra-Guerrero V, Sanhueza-Alvarado O, Cáceres-Espina M. Qualidade de vida de pessoas em hemodiálise crônica: relação com variáveis sociodemográficas, médico-clínicas e de laboratório. Rev Latinoam Enferm. 2012; 20(5):09 telas.

15. Kharame ZT, Zamanian H, Foroozanfar $\mathrm{S}$, Afsahi S. Religious wellbeing as a predictor for quality of life in iranian hemodialysis patients. Glob J Health Sci. 2014; 6(4):261-69.

16.Galvão TF, Pereira MG. Avaliação da qualidade da evidência de revisões sistemáticas. Epidemiol Serv Saúde. 2015; 24(1):173-75.

17.Kusumota L, Marques S, Haas VJ, Rodrigues RAP. Adultos e idosos em hemodiálise: avaliação da qualidade de vida relacionada à saúde. Acta Paul Enferm. 2008; 21(n esp):152-59.

18. Grasseli CSM, Chaves EC, Simão TP, Botelho PB, Silva RR. Avaliação da qualidade de vida dos pacientes submetidos à hemodiálise. Rev Bras Clin Med. 2012; 10(6):503-07. 
19.Cunha MS, Andrade V, Guedes CAV, Meneghetti CHZ, Aguiar AP, Cardoso AL. Avaliação da capacidade funcional e da qualidade de vida em pacientes renais crônicos submetidos a tratamento hemodialítico. Fisioter Pesqui. 2009; 16 (2):155-60.

20.Mortari DM, Menta M, Scapini KB, Rockembach CWF, Duarte A, Leguisamo CP. Qualidade de vida de indivíduos com doença renal crônica terminal submetidos à hemodiálise. Sci Méd. 2010; 20(2):156-60.

21.Silva KAL, Cargnin MCS, Ventura J, Paula SF, Groos JV. Qualidade de vida de pacientes com insuficiência renal em tratamento hemodialítico. Rev Enferm UFPE on line. [Internet]. 2017 [citado em 23 abr 2018]; 11(11):4663-70. Disponível em: https://periodicos.ufpe.br/revistas/revist aenfermagem/article/view/231207/2521 0

22.Liberati A, Altman DG, Tetzlaff J, Mulrow C, Gøtzsche PC, Ioannidis JPA, et al. The PRISMA statement for reporting systematic reviews and metaanalyses of studies that evaluate health care interventions: explanation and elaboration. J clin epidemiol. [Internet]. 2009 Jul [citado em 23 abr 2018]; 62(10):e1-e34. Disponível em: https://www.jclinepi.com/article/S08954356(09)00180-2/pdf

23. Pagels AA, Soderkvist BK, Medin C. Health-related quality of life in different stages of chronic kidney disease and at initiation of dialysis treatment. Health Qual Life Outcomes. 2012; 10:71.

24. Kalender B, Ozdemir AC, Dervisoglu E, Ozdemir O. Quality of life in chronic kidney disease: effects of treatment modality, depression, malnutrition and inflammation. Int J Clin Pract. 2007; 61 (4):569-6.

25.Ikonomou M, Skapinakis P, Balafa O, Eleftheroudi M, Damigos D, Siamopoulos KC. The impact of socioeconomic factors on quality of life of patients with chronic kidney disease in Greece. J Ren Care. 2015; 41(4):2396.

26. Pereira BS, Fernades NS, Melo NP, Abrita R, Grincenkov FRS, Fernandes NMS. Beyond quality of life: a cross sectional study on the mental health of patients with chronic kidney disease undergoing dialysis and their caregivers. Health Qual Life Outcomes [Internet]. 2017 [citado em 23 abr 2018]; 15(1):74. Disponível em: https://www.ncbi.nlm.nih.gov/pmc/artic les/PMC5392976/pdf/12955_2017_Arti cle_646.pdf

27.Lee YJ, Kim MS, Cho S, Kim SR. Association of depression and anxiety with reduced quality of life in patients with predialysis chronic kidney disease. Int J Clin Pract. 2013; 67(4):363-68.

28.Frazão CMFQ, Ramos VP, Lira ALBC. Qualidade de vida de pacientes submetidos à hemodiálise. Rev Enferm. UERJ. 2011; 19(4):577- 82

29. Cordeiro JABL, Brasil VV, Silva AMTC, Oliveira LMA, Zatta LT, Silva ACCM. Qualidade de vida e tratamento hemodialítico: avaliação do portador de insuficiência renal crônica. Rev Eletrônica Enferm. 2009; 11(4):785-83.

RECEBIDO: $14 / 06 / 2018$ APROVADO: $17 / 09 / 2018$ PUBLICADO: $12 / 18$ 